\title{
Relative semisimpliziale Approximation
}

\author{
WERNER Bos zum Gedächtnis \\ Von \\ Rudolf Frimsch
}

Es sei $A \subset X$ ein Paar von semisimplizialen Mengen. Eine $A$ enthaltende Unterteilung $X^{\prime}$ von $X$ ist eine semisimpliziale Menge $X^{\prime}$ zusammen mit einem Homöomorphismus $h:\left|X^{\prime}\right| \rightarrow|X|$, derart, daß $\left|X^{\prime}\right|$ als Unterteilung von $|X|$ im Sinne von [7] $\S 9$ aufgefaßt werden kann, $A$ semisimpliziale Teilmenge von $X^{\prime}$ ist und gilt

$$
h /|A|=(|A| \subset|X|) \text {. }
$$

Satz 1. Sei $A \subset X$ ein Paar semisimplizialer Mengen mit endlichem $\left.{ }^{1}\right) X$; ferner se $i$ $Y$ eine beliebige semisimpliziale Menge und $f:|X| \rightarrow|Y|$ eine stetige Abbildung mit

$$
f /|A|=|g|
$$

für eine semisimpliziale Abbildung $g: A \rightarrow Y$. Dann gibt es eine $A$ enthaltende Unterteilung $X^{\prime}$ von $X$ und eine semisimpliziale Abbildung $f^{\prime}: X^{\prime} \rightarrow Y$ mit

$$
\left|f^{\prime}\right| \simeq f \text { rel. }|A|
$$

Für simpliziale Komplexe findet sich dieser Satz z.B. in [8]; Rourke und Sanderson haben eine entsprechende Aussage für simpliziale Mengen ${ }^{2}$ ) bewiesen ([6] Theorem 5.1). Die semisimpliziale Approximation wurde z.T. bereits von Kan in [4] behandelt (vgl. dazu den Hinweis nach Satz 3).

Aussagen über die Existenz " $A$ enthaltender Unterteilungen von $X$ " liefert

Satz 2. Seien $\mathrm{n}$ eine natürliche Zahl und das Quadrat

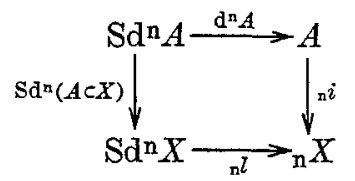

ein Pushout. Dann gibt es einen Homöomorphismus ${ }_{\mathrm{n}} h:\left|{ }_{\mathrm{n}} X\right| \rightarrow|X|$ mit

$$
{ }_{\mathrm{n}} h \simeq\left|{ }_{\mathrm{n}} r\right| \operatorname{rel} .|A| \text {, }
$$

derart, daß $\left.\right|_{\mathrm{n}} X \mid$ als Unterteilung von $|X|$ aufgefaßt werden kann.

1) Da die Inklusion $\left|A \cup X^{n}\right| \subset|X|$ für jedes $n$ die Homotopieerweiterungseigenschaft ([1], 1.2) hat, ergibt ein leichter Induktionsschluß, daß die Behauptung von Satz 1 auch für unendliche $X$ gilt.

2) Zur Terminologie vgl. [2], in [6] heißen die simplizialen Mengen „, 4 -sets ${ }^{c 4}$. 
(Hier bezeichnet $\mathrm{Sd}^{\mathrm{n}}$ die in [4] $\S 7$ definierte $\mathrm{n}$-fache Normalunterteilung und $\mathrm{d}^{\mathrm{n}}: \mathrm{Sd}^{\mathrm{n}} \rightarrow \mathrm{Id}$ die zugehörige natürliche Transformation; ${ }_{\mathrm{n}} r:{ }_{\mathrm{n}} X \rightarrow X$ ist die durch die Gleichung

$$
{ }_{\mathrm{n}} r \circ \mathrm{n} l=\mathrm{d}^{\mathrm{n}} X
$$

eindeutig bestimmte semisimpliziale Abbildung.)

Wir beweisen diesen Satz durch vollständige Induktion nach $\mathrm{n}$.

Sei zunächst $\mathrm{n}=1$. Für jedes $x \in X_{q}$ und jedes $q$ bezeichne $c_{x}: \Delta[q] \rightarrow X$ die charakteristische Abbildung und $l_{x}: \Delta_{q} \rightarrow \Delta_{x}$ den surjektiven Teil von $\left|{ }_{1} l_{\circ} \operatorname{Sd}^{\mathrm{n}} c_{x}\right|$. Wir definieren ferner Abbildungen $h_{x}: \Delta_{q} \rightarrow \Delta_{q}$ wie in [3] unter der Abänderung der Definition der Operatoren $\varrho_{j}$ (falls $x \mu_{j} \in A$ ): In [3] bezeichnet $\varrho_{j}$ den surjektiven Operator in der kanonischen Darstellung von $x \mu_{j}$. Ist nun $x \mu_{j} \in A$, so setzen wir statt dessen $\varrho_{j}:=\left(\left[\operatorname{dim} \mu_{j}\right] \rightarrow[0]\right)$; entsprechend sind natürlich die $\varrho_{k j}$ und $\tilde{\varrho}_{k j} a b$ zuändern. Mit diesen Festsetzungen gilt Bedingung (A) aus [3] und Bedingung (B) aus [3] sinngemäß für nicht entartete $x$ mit $x \notin A$; man beweist das genauso wie in [3]. Ferner ist aber die Transformation $h_{x} \circ l_{x}^{-1}$ einwertig und damit stetig; insbesondere ist für $x \in A$

$$
h_{x} \circ l_{x}^{-1}=\mathrm{id} .
$$

Diese Aussagen zusammen liefern, daß die Familie der Abbildungen $h_{x} \circ l_{x}^{-1}$ einen Homöomorphismus $1 h:\left|{ }_{1} X\right| \rightarrow|X|$ induziert. Die gesuchte Homotopie erhält man nun noch aus der Tatsache, daß auch die Transformationen $\left((1-t) h_{x}+t d_{x}\right) \circ l_{x}^{-1}$ für alle $t \in[0,1]$ einwertig und damit stetig sind.

Zum Schluß von $n$ auf $n+1$ betrachten wir das Diagramm

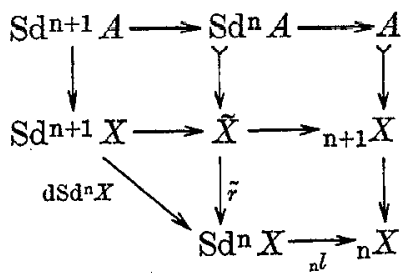

Es ist so konstruiert, daß die drei Quadrate Pushouts sind. Der bereits bewiesene Fall $\mathrm{n}=1$ zeigt, daß $\tilde{X}$ eine $\operatorname{Sd}^{n} A$ enthaltende Unterteilung von $\operatorname{Sd}^{\mathbf{n}} X$ ist, vermöge eines Homöomorphismus $\tilde{h}:|\tilde{X}| \rightarrow\left|\operatorname{Sd}^{n} X\right|$, für den gilt:

$$
\hbar \simeq|\tilde{r}| \operatorname{rel} .\left|\operatorname{Sdn}^{n} A\right| \text {. }
$$

Sei $\tilde{h}_{t}$ eine solche Homotopie mit $\tilde{h}_{0}=\tilde{h}$ und $\tilde{h}_{1}=|\tilde{r}|$. Wir definieren nun für alle $t \in[0,1]$ stetige Abbildungen $g_{t}$ durch die Forderung, daß die Quadrate

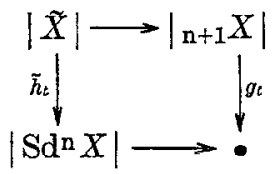

Pushouts sind. Aus der Tatsache, daß dann für jedes $t$ das äußere Rechteck in dem 
Diagramm

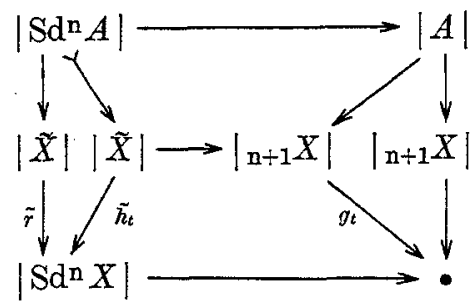

ein Pushout ist, ergibt sich, daß die $g_{t}$ alle das gleiche Ziel $|\mathrm{n} X|$ haben und, da sie stetig von $t$ abhängen, eine Homotopie rel. $|A|$ bilden. Da $\tilde{h}_{0}$ ein Homöomorphismus ist, ist auch $g_{0}$ ein Homöomorphismus und die Zusammensetzung ${ }_{\mathrm{n}+1} h:={ }_{\mathrm{n}} h \circ g_{0}$ ist ein Homöomorphismus der gewünschten Art.

Zum Beweis von Satz 1 benötigen wir nun noch

Satz 3. Unter den Voraussetzungen von Satz 1 gibt es eine natürliche Zahl $\mathbf{n}$ und eine semisimpliziale Abbildung $f^{\prime \prime}: \mathrm{Sd}^{\mathrm{n}} X \rightarrow Y$ mit

$$
\left|f^{\prime \prime}\right| \simeq f \circ\left|\mathrm{d}^{\mathrm{n}} X\right| \text { rel. }\left|\operatorname{Sd}^{\mathrm{n}} A\right| \text {. }
$$

Für $A=\emptyset$ ist das einer der Kanschen Approximationssätze (Theorem (8.5) in [4]). Wir führen den Beweis durch Verfeinerung der Argumente in [4] und verwenden dabei die dortige Notation. Auf Grund des Satzes in II 5.8 von [5] gibt es jedenfalls eine semisimpliziale Abbildung $\tilde{F}: X \rightarrow \mathrm{Ex}^{\infty} Y$ mit

$$
|\tilde{F}| \simeq\left|\mathrm{e}^{\infty} Y\right| \circ f \text { rel. }|A| \text {. }
$$

Aus der Endlichkeit von $X$ folgt nun ähnlich wie in [4], p. 463, die Existenz einer natürlichen Zahl $\mathrm{n}$ und einer semisimplizialen Abbildung $F: X \rightarrow \operatorname{Ex}^{\mathrm{n}} Y$ mit

$$
|F| \simeq\left|\mathrm{e}^{\mathrm{n}} Y\right| \circ f \mathrm{rel} .|A| \text {. }
$$

Komposition mit $\mathrm{d}^{\mathrm{n}} X$ liefert jetzt

$$
\left|F \circ \mathrm{d}^{\mathrm{n}} X\right| \simeq\left|\mathrm{e}^{\mathrm{n}} Y\right| \circ f \circ\left|\mathrm{d}^{\mathrm{n}} X\right| \operatorname{rel} .\left|\operatorname{Sd}^{\mathrm{n}} A\right| \text {. }
$$

Für die linke Seite können wir wegen der Natürlichkeit von $d^{n}$ schreiben

$$
F \circ \mathrm{d}^{\mathrm{n}} X=\mathrm{d}^{\mathrm{n}} \operatorname{Ex}^{\mathrm{n}} Y \circ \operatorname{Sd}^{\mathrm{n}} F \text {. }
$$

Nun bezeichne $\varepsilon Y: \operatorname{Sd}^{\mathbf{n}} \operatorname{Ex}^{\mathrm{n}} Y \rightarrow Y$ die Coeins der Adjunktion $\operatorname{Ex}^{\mathbf{n}} \rightarrow \operatorname{Sd}^{\mathbf{n}}$, d.h. die semisimpliziale Abbildung, die unter dem Adjunktionsisomorphismus in die Identität von $\operatorname{Ex}^{\mathrm{n}} Y$ übergeht. Weil $\mathrm{d}^{\mathrm{n}} Y$ unter dem Adjunktionsisomorphismus in $\mathrm{e}^{\mathrm{n}} Y$ übergeht ([4] Lemma 7.2) gilt

$$
\mathrm{d}^{\mathrm{n}} Y=\varepsilon Y \circ \mathrm{Sd}^{\mathrm{n}} \mathrm{e}^{\mathrm{n}} Y
$$

und daher wegen der Natürlichkeit von $d^{n}$

$$
\mathrm{d}^{\mathrm{n}} \operatorname{Ex}^{\mathrm{n}} Y \circ \operatorname{Sd}^{\mathrm{n}} \mathrm{e}^{\mathrm{n}} Y=\mathrm{e}^{\mathrm{n}} Y \circ \mathrm{d}^{\mathrm{n}} Y=\mathrm{e}^{\mathrm{n}} Y \circ \varepsilon Y \circ \operatorname{Sd}^{\mathrm{n}} \mathrm{e}^{\mathrm{n}} Y .
$$

Aus Lemma (7.4) und Lemma (7.5) in [4] folgt, daß $\left|\operatorname{Sd}^{\mathbf{n}} e^{n} Y\right|$ eine Homotopie- 
äquivalenz ist. $\mathrm{Da} \operatorname{Sd}^{\mathrm{n}} \mathrm{e}^{\mathrm{n}} Y$ außerdem injektiv und damit $\left|\mathrm{Sd}^{\mathrm{n}} \mathrm{e}^{\mathrm{n}} Y\right|$ eine Cofaserung ist, ist $\left|\mathrm{Sd}^{\mathrm{n}} \mathrm{e}^{\mathrm{n}} Y\right|$ eine Homotopieäquivalenz unter $\left|\mathrm{Sd}^{\mathrm{n}} Y\right|([1],(2.29))$, woraus folgt

$$
\left|\mathrm{d}^{\mathrm{n}} \operatorname{Ex}^{\mathrm{n}} Y\right| \simeq\left|\mathrm{e}^{\mathrm{n}} Y\right| \circ|\varepsilon Y| \operatorname{rel} .\left|\operatorname{Sd}^{\mathrm{n}} Y\right| .
$$

Komposition mit $\left|\operatorname{Sd}^{\mathfrak{n}} F\right|$ liefert nun

$$
\left|\mathrm{d}^{\mathrm{n}} \operatorname{Ex}^{\mathrm{n}} Y \circ \operatorname{Sd}^{\mathrm{n}} F\right| \simeq\left|\mathrm{e}^{\mathrm{n}} Y \circ \varepsilon Y \circ \operatorname{Sd}^{\mathrm{n}} F\right| \operatorname{rel} .\left|\operatorname{Sd}^{\mathrm{n}} A\right| .
$$

(15), (16) und (20) zusammen ergeben

$$
\left|\mathrm{e}^{\mathrm{n}} Y\right| \circ\left|\varepsilon Y \circ \operatorname{Sd}^{\mathrm{n}} F\right| \simeq\left|\mathrm{e}^{\mathrm{n}} Y\right| \circ f \circ\left|\mathrm{d}^{\mathrm{n}} X\right| \operatorname{rel} .\left|\operatorname{Sd}^{\mathrm{n}} A\right| \text {. }
$$

Weil $\left|\mathrm{e}^{\mathrm{n}} Y\right|$ eine Homotopieäquivalenz unter $|Y|$ ist ([1], (2.29)), folgt

$$
\left|\varepsilon Y \circ \operatorname{Sd}^{\mathrm{n}} F\right| \simeq f \circ\left|\mathrm{d}^{\mathrm{n}} L\right| \operatorname{rel} .\left|\mathrm{Sd}^{\mathrm{n}} A\right| \text {. }
$$

Also ist $f^{\prime \prime}:=\varepsilon Y \circ \mathrm{Sd}^{\mathrm{n}} F$ die gesuchte Approximation zu $f$.

Wir kommen nun zum Beweis von Satz 1. Das gesuchte $f^{\prime}$ ist durch das Diagramm

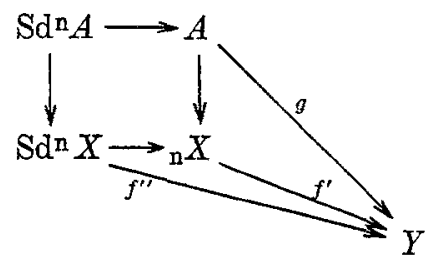

gegeben und eindentig bestimmt ( $n, f^{\prime \prime}$ wie in Satz 3). Da die geometrische Realisierung Pushouts erhält, liefert die Homotopie in Satz 3 eine Homotopie

$$
\left.\left|f^{\prime}\right| \simeq f \circ\right|_{\mathrm{n}} r|\operatorname{rel} .| A \mid
$$

Zusammen mit (5) ergibt das die Behauptung (3).

\section{Literaturverzeichnis}

[1] T. том Dieck, K. H. Kamps und D. PuPpe, Homotopietheorie. Lecture Notes in Math. 78, Berlin-Heidelberg-New York 1970.

[2] R. Frimscr, Simpliziale und semisimpliziale Mengen. Bull. Acad. Polon. Sci. Sér. Sci. Math. Astronom. Phys. 20, 159-168 (1972).

[3] R. Fritsch und D. PUPPE, Die Homöomorphie der geometrischen Realisierungen einer semisimplizialen Menge und ihrer Normalunterteilung. Arch. Math. 18, 508-512 (1967).

[4] D. M. KaN, On c.s.s. complexes. Amer. J. Math. 79, 449-476 (1957).

[5] K. Lamotke, Semisimpliziale algebraische Topologie. Berlin-Heidelberg-New York 1968.

[6] C. P. Rotre and B. J. SANDERSor, $\Delta$-sets I: homotopy theory. Quart. J. Math. Oxford (2), 22, 321-338 (1971).

[7] J. H. C. Whitehead, Combinatorial homotopy, I. Bull. Amer. Math. Soc. 55, 213-245 (1949).

[8] E. C. Zemmann, Relative simplicial approximation. Proc. Cambridge Phil. Soc. 60, 39-43 (1964).

Eingegangen am 8. 12. 1972

Anschrift des Autors:

Rudolf Fritsch

Fachbereich Mathematik, Universität Konstanz, D-775 Konstanz 